\title{
Sexualität bei Menschen mit Autismus-Spektrum-Störung und ihre Bedeutung für die forensische Psychiatrie und Psychotherapie
}

\author{
Daniel Turner ${ }^{1}$ Daniel Schöttle ${ }^{2}$ Peer Briken ${ }^{3}$ \\ Eingegangen: 1. September 2020 / Angenommen: 1. Oktober 2020 / Online publiziert: 17. November 2020 \\ ○ Der/die Autor(en) 2020
}

\section{Zusammenfassung}

Menschen mit einer Autismus-Spektrum-Störung zeigen die gesamte Vielfalt sexueller Fantasien und sexuellen Verhaltens ebenso wie ihre nichtbetroffenen Peers. Dennoch finden sich auch einige Besonderheiten in sexuellem Erleben und Verhalten bei Menschen mit einer Autismus-Spektrum-Störung, die größtenteils auf die störungsspezifischen Symptome zurückzuführen sind. Hierzu zählen eine höhere Diversität bezüglich der eigenen sexuellen Orientierung sowie des eigenen Geschlechtsrollenverständnisses, aber auch Probleme im Hinblick auf das Eingehen von festen Partnerschaften oder im Hinblick auf die eigene sexuelle Funktionsfähigkeit. Darüber hinaus finden sich bei manchen Personen mit einer Autismus-Spektrum-Störung (insbesondere bei Männern) Hinweise auf das Vorliegen hypersexueller Verhaltensweisen sowie paraphiler sexueller Fantasien und Verhaltensweisen. Bisher existieren keine empirischen Befunde, die nahelegen, dass eine Autismus-Spektrum-Störung einen Risikofaktor für allgemeine oder sexuelle Straffälligkeit darstellt. Basierend auf Fallstudien wurden jedoch einige Erklärungsansätze publiziert, die beschreiben, wie in einigen wenigen Fällen die autismusspezifische Symptomatik mit zu der Begehung einer Straftat beigetragen haben könnte, was auch bei der Schuldfähigkeitsbegutachtung Berücksichtigung finden könnte. Hierzu zählen eine fehlende Opferempathie, das Vorliegen kognitiver Verzerrungen oder defizitäre Kommunikations- und Interaktionsfertigkeiten. Straftäter mit einer Autismus-Spektrum-Störung sollten individuell auf sie zugeschnittenen psycho- und pharmakotherapeutischen Interventionen zugeführt werden, um eine erneute Straffälligkeit im besten Falle zu verhindern.

Schlüsselwörter Asperger $\cdot$ Sexualstraftaten $\cdot$ Hypersexualität $\cdot$ Paraphilien $\cdot$ Sexuelle Orientierung

Dr. rer. biol. hum. Dr. med. Daniel Turner

daniel.turner@unimedizin-mainz.de

Prof. Dr. med. Peer Briken

peer.briken@uke.de

1 Klinik für Psychiatrie und Psychotherapie, Universitätsmedizin Mainz, Mainz, Deutschland
2 Klinik für Psychiatrie und Psychotherapie, Universitätsklinikum Hamburg-Eppendorf (UKE), Hamburg, Deutschland

3 Institut für Sexualforschung, Sexualmedizin und Forensische Psychiatrie, Universitätsklinikum Hamburg-Eppendorf (UKE), Hamburg, Deutschland 


\title{
Sexuality in people with autism spectrum disorder and its importance for forensic psychiatry and psychotherapy
}

\begin{abstract}
Individuals with autism spectrum disorder (ASD) show the whole range of sexual fantasies and sexual behavior just like their non-affected counterparts. Nevertheless, individuals with ASD show some peculiarities concerning sexual experiences and sexual behavior that can mainly be traced back to the disorder-inherent symptoms. Among these peculiarities are an increased diversity concerning their own sexual orientation and their understanding of gender roles as well as problems concerning the initiation of romantic or sexual partnerships or problems with sexual functioning. Furthermore, some individuals with ASD (especially men) show signs of hypersexual and/or paraphilic fantasies and behavior. So far, there exist no empirical results that would suggest that ASD is a specific risk factor for general or sexual offending; however, based on case studies some authors outlined in how far the disorder-inherent symptoms could have contributed to offending behavior in special cases, which could also be taken into account when assessing criminal responsibility. These include reduced victim empathy, cognitive distortions or deficits in communication or social interaction abilities. Specialized psychotherapeutic and pharmacotherapeutic interventions should be provided for individuals with ASD in order to prevent criminal recidivism.
\end{abstract}

Keywords Asperger $\cdot$ Sex offences $\cdot$ Hypersexuality $\cdot$ Paraphilias $\cdot$ Sexual orientation

\section{Einleitung}

Bei manchen Menschen mit einer Autismus-Spektrum-Störung finden sich Besonderheiten im Verhalten, in den Emotionen und Kognitionen, die einen nennenswerten Einfluss auf die erlebte und gelebte Sexualität entfalten können und die somit auch in der Begutachtung, Therapie und Prävention von Sexualdelikten Berücksichtigung finden sollten. $\mathrm{Zu}$ den Kernsymptomen einer Autismus-Spektrum-Störung gehören eine qualitative Beeinträchtigung der gegenseitigen sozialen Interaktion, qualitative Auffälligkeiten in der Kommunikation und begrenzte, repetitive und stereotype Verhaltensmuster, Interessen und Aktivitäten (American Psychiatric Association 2013). In Abhängigkeit des Schweregrades dieses Symptomkomplexes sowie des Alters bei Erstmanifestation unterscheidet die ICD-10 (International Classification of Diseases-10th revision) zwischen frühkindlichem Autismus (F84.0), atypischem Autismus (F84.1) und dem Asperger-Syndrom (F84.5) (World Health Organization 1993). Während die Symptome beim frühkindlichen Autismus bereits vor dem dritten Lebensjahr auftreten und häufig mit einer beträchtlichen Entwicklungsverzögerung einhergehen, zeigen sich Symptome beim atypischen Autismus entweder erst nach dem dritten Lebensjahr, oder die Diagnosekriterien für einen frühkindlichen Autismus sind nicht vollständig erfüllt. Bei ca. 30-50\% der Betroffenen liegt zudem eine eingeschränkte intellektuelle Leistungsfähigkeit vor (World Health Organization 1993). Bei Personen mit einem Asperger-Syndrom findet sich im Gegensatz zum frühkindlichen und atypischen Autismus keine Verzögerung der Sprach- und kognitiven Entwicklung sowie eine teils augenscheinlich geringer ausgeprägte Symptomatik, was dazu führt, dass das Asperger-Syndrom häufig erst im Adoleszenten- oder im Erwachsenenalter mit dem Ansteigen der sozialen Anforderungen diagnostiziert wird (World Health Organization 1993). Bei genauerer Betrachtung der sozialen Entwicklung eines Betroffenen lassen sich in der Retrospektive spezifische Auffälligkeiten häufig bereits in der Kindheit finden, die zu diesem Zeitpunkt jedoch noch nicht $\mathrm{zu}$ einer von außen erkennbaren wesentlichen Beeinträchtigung geführt haben oder die z.B. durch Erfüllen von Rollenklischees (,das zurückhaltende schüchterne Mädchen") übersehen wurden. In der ICD-11 wird diese diagnostische Dreiteilung in Anlehnung an das DSM-5 (Diagnostic and Statistical Manual of Mental Disorders-5th revision) verlassen, und alle Störungsbilder werden unter dem Begriff der Autismus-Spektrum-Störung zusammengefasst. Des Weiteren sollen innerhalb dieser neuen diagnostischen Kategorie Unterteilungen bezüglich des Vorliegens einer Intelligenzminderung sowie einer Sprachentwicklungsverzögerung vorgenommen werden können (Matthies und Frauenknecht 2019).

Es findet sich eine Lebenszeitprävalenz für die Autismus-Spektrum-Störungen von etwa $1 \%$, wobei Männer im Verhältnis 2-3:1 deutlich häufiger betroffen sind als Frauen (Fombonne 2009). Häufig auftretende komorbide psychische Erkrankungen sind das Aufmerksamkeitsdefizit-/ Hyperaktivitätssyndrom (ADHS), Tic-Störungen, Zwangsstörungen, depressive Syndrome und Persönlichkeitsstörungen (v.a. schizoide und schizotype Persönlichkeitsstörungen) (Matthies und Frauenknecht 2019). Diese häufig anzutreffenden komorbiden Erkrankungen weisen zahlreiche überschneidende Symptome mit Störungen aus dem $\mathrm{Au}$ tismusspektrum auf, sodass die Zuordnung der vorhandenen Symptome zu den einzelnen Störungsbildern eine wesentliche diagnostische Herausforderung darstellt und um- 
fassende Kenntnis der diagnostischen Kriterien der einzelnen Störungsbilder voraussetzt. Im besten Falle sollte eine Diagnosestellung insbesondere im Erwachsenenalter und bei Vorliegen einer durchschnittlichen intellektuellen Leistungsfähigkeit in einem spezialisierten Zentrum unter Einbezug von standardisierten Testverfahren (z. B. der Diagnostischen Beobachtungsskala für Autistische Störungen [ADOS; Poustka et al. 2015] oder dem Diagnostischen Interview für Autismus-Revidiert [ADI-R; Bölte et al. 2006]) und Hinzuziehen fremdanamnestischer Informationen stattfinden. Da sich die Behandlung der einzelnen Störungsbilder trotz ihrer Gemeinsamkeiten teilweise bedeutend unterscheiden kann, ist eine präzise diagnostische Einordnung umso wichtiger.

\section{Sexualität und Autismus-Spektrum- Störungen}

Gut ausgeprägte Fähigkeiten zur sozialen Kommunikation, Interaktion und zur Emotionsregulation, das Teilen von Interessen und Aktivitäten mit anderen sowie gut ausgeprägte Theory-of-Mind-Fähigkeiten stellen wesentliche Voraussetzungen für eine zufriedenstellende und respektvoll gestaltete Sexualität dar. Da sich bei Menschen mit einer Autismus-Spektrum-Störung Auffälligkeiten in all diesen Bereichen finden können, existierten lange Zeit zahlreiche Vorurteile bezüglich der Sexualität von Menschen mit einer Autismus-Spektrum-Störung. Beispielsweise wurde angenommen, dass Menschen mit einer Autismus-SpektrumStörung wenig bis kein Interesse an intimen Beziehungen zeigen, oder dass die eigene Sexualität hauptsächlich in Form von Masturbation ausgelebt wird (Koller 2000; Konstantareas und Lunsky 1997). In den letzten Jahren konnte in zahlreichen empirischen Arbeiten jedoch gezeigt werden, dass entgegen dieser früheren Vorstellungen, Menschen mit einer Autismus-Spektrum-Störung durchaus Interesse an partnerschaftlicher sowie Solosexualität zeigen und sich die gesamte Bandbreite sexueller Verhaltensweisen ebenso wie bei nichtbetroffenen Menschen finden lässt (Strunz et al. 2017; Turner et al. 2017). Dennoch lässt sich auch der Einfluss der krankheitsspezifischen Verhaltensauffälligkeiten auf das sexuelle Verhalten nicht ignorieren. Im Folgenden soll eine Übersicht über die Besonderheiten der sexuellen Vorlieben, Fantasien und Verhaltensweisen von Menschen mit einer Autismus-Spektrum-Störung gegeben werden. Abschließend wird die Bedeutung einer Autismus-Spektrum-Störung im Zusammenhang mit (sexueller) Straffälligkeit diskutiert. Der Fokus liegt hierbei auf Personen mit einer normalen intellektuellen Leistungsfähigkeit (Asperger-Syndrom oder hochfunktionalem Autismus).

\section{Sexuelle Orientierung und eigene Geschlechtswahrnehmung}

Mittlerweile existieren zahlreiche Hinweise, dass sich Menschen mit einem Asperger-Syndrom im Vergleich zur Allgemeinbevölkerung häufiger als nicht-heterosexuell beschreiben. Zwar fehlen weitestgehend repräsentative Angaben über die Verteilung und Häufigkeit der unterschiedlichen sexuellen Orientierungen in der Allgemeinbevölkerung, es wird jedoch geschätzt, dass zwischen 5 und $10 \%$ der Männer und Frauen sich als nicht-heterosexuell beschreiben, wobei hierzu homo- und bisexuelle Personen zählen, sowie Menschen, die sich keiner dieser drei Kategorien zugehörig fühlen (Shaeer und Sheer 2015). Demgegenüber wird die Prävalenz einer nicht-heterosexuellen Orientierung bei Menschen mit einer Autismus-Spektrum-Störung auf etwa 15-35\% geschätzt (Hellemans et al. 2007; Turner et al. 2017). Hierbei scheinen Frauen mit einer Autismus-Spektrum-Störung signifikant häufiger über eine nicht-heterosexuelle Orientierung zu berichten als Männer (Turner et al. 2017). Es wird angenommen, dass sich Menschen mit einer Autismus-Spektrum-Störung in ihrem Verhalten weniger an sozialen Normen orientieren und sich somit auch toleranter gegenüber nicht-heterosexuellen Partnerschaften zeigen. Die Partnerwahl hängt in diesem Fall in höherem Ausmaß von dem genuinen Interesse am Gegenüber ab, unabhängig von dessen oder deren Geschlecht (Turner et al. 2017).

In einer systematischen Übersichtsarbeit konnte gezeigt werden, dass sich sowohl unter Kindern und Jugendlichen als auch unter Erwachsenen mit einer Geschlechtsdysphorie eine erhöhte Prävalenz einer komorbiden Autismus-Spektrum-Störung findet, als dies im Vergleich zur Allgemeinbevölkerung zu erwarten wäre (Glidden et al. 2016). Mögliche Erklärungsansätze für diesen Zusammenhang umfassen die Theorie eines besonders männlich ausgebildeten Gehirns, erhöhte pränatale Androgen-Serum-Spiegel sowie erhöhte Androgen-Serum-Spiegel in der Kindheit und Adoleszenz (bei Personen, denen ein weibliches Geburtsgeschlecht zugewiesen wurde), Beeinträchtigungen in der Mutter-KindBindung, die geringe persönliche Bedeutung der traditionellen Geschlechterrollen bei der Definition des eigenen Selbst und eine häufig gering ausgeprägte Orientierung an sozialen Normvorstellungen (Glidden et al. 2016). Es finden sich aber auch Befunde, die gegen den oben postulierten Zusammenhang sprechen, sodass es bislang nicht als gesichert angesehen werden sollte, dass sich in Populationen von Erwachsenen mit einer Geschlechtsdysphorie eine erhöhte Prävalenz von Autismus-Spektrum-Störungen finden lässt (Oien et al. 2018). 


\section{Sexuelle und romantische Beziehungen}

Wie bereits weiter oben dargestellt, existierte lange Zeit die Überzeugung, dass Menschen mit einer Autismus-Spektrum-Störung nur wenig Interesse an sexuellen und romantischen Beziehungen zeigen (Koller 2000; Konstantareas und Lunsky 1997). Sowohl Männer als auch Frauen mit einer Autismus-Spektrum-Störung zeigen demgegenüber ein deutliches Interesse an und den Wunsch nach einer festen Partnerschaft, und in den verschiedenen Untersuchungen waren es in der Regel weniger als 10\% der Befragten, die eine partnerschaftliche Beziehung nicht für sich in Betracht zogen (z. B. Strunz et al. 2017). Trotzdem befinden sich Menschen mit einer Autismus-Spektrum-Störung seltener in einer festen Beziehung als Menschen aus der Allgemeinbevölkerung ohne psychische Erkrankung (Schöttle et al. 2017). Dies könnte eine Folge der letztlich doch eingeschränkten Fähigkeiten in der sozialen Interaktion und Kommunikation sein, die das Eingehen und Aufrechterhalten einer Partnerschaft erschweren könnten. Daher verwundert es auch nicht, dass sich häufig Beziehungen finden, in denen beide Personen autistische Züge aufweisen und diese ihre Beziehung als glücklicher einschätzen als Personen in Beziehungen, in denen nur ein Partner autistische Wesenszüge zeigt (Strunz et al. 2017).

Es finden sich deutliche Geschlechtsunterschiede in der Beziehungshäufigkeit von Menschen mit einer AutismusSpektrum-Störung. Während sich betroffene Männer häufiger eine feste Beziehung wünschen, im Vergleich zu Frauen mit einer Autismus-Spektrum-Störung, befinden sich Frauen deutlich häufiger in einer festen Partnerschaft (Turner et al. 2017). Die Einschränkungen in den sozialen Interaktions- und Kommunikationsfähigkeiten sind bei Frauen mit einer Autismus-Spektrum-Störung meist weit weniger intensiv ausgeprägt, und Frauen besitzen teilweise bessere Coping-Strategien, um die vorhandenen Defizite im täglichen Leben auszugleichen, wie beispielsweise eine ausgeprägte Fähigkeit, soziale Verhaltensweisen der nicht an einer Autismus-Spektrum-Störung erkrankten Peers nachzuahmen (,social mimicry“) (Dean et al. 2017). Dies könnte es Frauen mit einer Autismus-Spektrum-Störung erleichtern, eine sexuelle Beziehung einzugehen.

\section{Sexuelle Verhaltensweisen und sexuelle Störungen}

Insgesamt berichteten sowohl Männer als auch Frauen mit einer Autismus-Spektrum-Störung in mehreren Studien über weniger regelmäßigen Geschlechtsverkehr im Vergleich zu Personen aus der Allgemeinbevölkerung (Byers et al. 2013a, 2013b; Schöttle et al. 2017). Dies könnte als eine direkte Folge der geringeren Anzahl an Personen mit einer Autismus-Spektrum-Störung, die in einer festen Partnerschaft leben, angesehen werden. Darüber hinaus finden sich sowohl bei Männern als auch bei Frauen mit einer Autismus-Spektrum-Störung häufiger Hinweise auf sexuelle Funktionsstörungen, die ebenfalls das partnerschaftliche Sexualleben beeinträchtigen könnten (Turner et al. 2019). Hierbei scheint die Häufigkeit sexueller Funktionsstörungen besonders bei den weiblichen Betroffenen erhöht zu sein und schließt alle Bereiche des sexuellen Reaktionszyklus ein (z.B. vermindertes sexuelles Verlangen, verminderte sexuelle Erregung, verminderte Orgasmushäufigkeit, verminderte allgemeine sexuelle Zufriedenheit sowie mehr sexuelle Schmerzen) (Turner et al. 2019). Zur Erklärung dieses Zusammenhangs wurde angeführt, dass sich insbesondere die ausgeprägten Hypo- und/oder Hypersensibilitäten in verschiedenen sensorischen Bereichen, die sich regelhaft bei Menschen mit einer Autismus-SpektrumStörung finden lassen, für die Entstehung der sexuellen Funktionsstörungen verantwortlich zeichnen könnten. Während bestimmte Reize, z.B. olfaktorische Reize, besonders intensiv wahrgenommen werden, werden andere Reize, z.B. taktile Reize, nur sehr schwach wahrgenommen, wobei die Ausprägung individuell ganz verschieden sein kann (Pellicano 2013). Des Weiteren könnten die Defizite in der sozialen Kommunikation und Interaktion nicht nur das Führen einer festen Partnerschaft, sondern auch das Initiieren kurzer sexueller Kontakte erschweren.

Demgegenüber berichteten in mehreren Untersuchungen zumindest Männer mit einer Autismus-Spektrum-Störung über eine höhere Masturbationsfrequenz im Vergleich zu Männern aus der Allgemeinbevölkerung (Fernandes et al. 2016; Schöttle et al. 2017). Die Neigung zu repetitiven und stereotypen Interessen und Verhaltensweisen könnte hierzu beitragen. Diese Neigung ist bei Männern häufig deutlich stärker ausgeprägt, was auch hier den Geschlechtsunterschied erklären könnte. In seiner maximalen Ausprägung könnte die höhere Masturbationsfrequenz aufseiten der Männer mit einer Autismus-Spektrum-Störung bis hin zur Entwicklung hypersexuellen Verhaltens führen. Sind zusätzliche Kriterien wie ein persönlicher Leidensdruck aufseiten des Betroffenen zu finden, rechtfertigt dies die Diagnose einer hypersexuellen Störung oder nach der ICD11 einer ,Zwanghaften sexuellen Verhaltensstörung“. Hierbei kann sich das sexuelle Verhalten in jeder Art von Verhalten äußern, das bei der betroffenen Person zu sexueller Erregung führt und mit dem Ziel, einen Orgasmus $\mathrm{zu}$ erleben, durchgeführt wird (Turner und Briken 2017). In einer ersten eigenen Untersuchung mit 56 Männern mit einem Asperger-Syndrom und 56 Männern aus der Allgemeinbevölkerung (gematched nach Alter und Bildungsstand) berichteten Männer mit Asperger-Syndrom häufiger über hypersexuelle Verhaltensweisen. Während $30 \%$ der Männer mit einer Autismus-Spektrum-Störung über dem vorgeschlagenen „Cut-off“-Wert für eine hypersexuelle Störung des Hypersexual Behavior Inventory 
(Reid et al. 2011) lagen, waren dies nur 4\% der Männer aus der Allgemeinbevölkerung. Bei den 80 Studienteilnehmerinnen mit einer Autismus-Spektrum-Störung und der Allgemeinbevölkerung fanden sich dagegen keine Unterschiede in der selbst-berichteten Häufigkeit hypersexueller Verhaltensweisen (Schöttle et al. 2017).

Neben der ausgeprägten Neigung zu repetitivem Verhalten finden sich bei Menschen mit einer Autismus-SpektrumStörung, wie weiter oben bereits erwähnt, häufig auch ausgeprägte Hypo- oder Hypersensibilitäten in den verschiedenen Sinnessystemen. Die besonders intensive Wahrnehmung der sexuellen Stimulation (bis hin zur Förderung eines suchtartigen Charakters bezüglich sexueller Reize) ebenso wie eine nur schwache Wahrnehmung (besonders intensive und häufige sexuelle Stimulation wird benötigt, damit diese als ausreichend empfunden wird) könnten zu hypersexuellem Verhalten führen (Schöttle et al. 2017).

Aber nicht nur quantitativ, sondern auch qualitativ existieren Unterschiede bezüglich der Sexualität zwischen Personen mit und ohne Autismus-Spektrum-Störung. In der oben dargestellten Untersuchung berichteten wiederum Männer mit einer Autismus-Spektrum-Störung häufiger über paraphile sexuelle Fantasien, wie masochistische, sadistische, voyeuristische, frotteuristische sowie pädophile sexuelle Fantasien. Diese Unterschiede fanden sich überwiegend auf der Ebene sexueller Fantasien, während sich auf der Verhaltensebene keine Unterschiede zeigten (mit Ausnahme voyeuristischer und frotteuristischer Verhaltensweisen, die sich ebenso häufiger bei Männern mit einer Autismus-Spektrum-Störung fanden). Wiederum konnten bei Frauen mit und ohne Autismus-Spektrum-Störung keine diesbezüglichen Unterschiede gefunden werden (Schöttle et al. 2017). Es ist vorstellbar, dass die höhere Frequenz (hyper-)sexueller Verhaltensweisen zu einer Habituation bezogen auf sexuelle Reize führt, was immer ausgefallenere und spezifischere sexuelle Reize für eine ausreichende Erregung nötig machen könnte. Hinzu kommen noch die ohnehin überdurchschnittlich ausgeprägte Neigung zu repetitiven und stereotypen Verhaltensweisen sowie die Hypound Hypersensibilitäten, welche die Wahrscheinlichkeit der Entwicklung paraphiler sexueller Interessen in einem Individuum mit einer Autismus-Spektrum-Störung weiter erhöhen könnten (Schöttle et al. 2017).

\section{Bedeutung der Autismus-Spektrum- Störungen für die forensische Psychiatrie}

Es existieren bislang keine Hinweise darauf, dass sich in Straftäterpopulationen eine erhöhte Prävalenz von Menschen mit einer Autismus-Spektrum-Störung finden lässt (King und Murphy 2014; Mouridsen 2012; WoodburySmith et al. 2006). Auch innerhalb der Gruppe der Se- xualstraftäter findet man keine erhöhte Prävalenz einer Autismus-Spektrum-Störung, jedoch fehlen systematische Studien auch hierzu weitestgehend. Bislang existieren hauptsächlich Fallbeschreibungen, in denen das sexuell übergriffige Verhalten von ,einer fremden Person einen Kuss geben“ (Clements und Zarkowska 2000) bis hin zu einem sexuell gewalttätigen Übergriff reicht (Murrie et al. 2002). Die dargestellten Patienten zeigen des Weiteren ganz unterschiedliche kognitive Einschränkungen (Brendel et al. 2002; Chan und Saluja 2011), sodass der Versuch, haltbare Gemeinsamkeiten zwischen den einzelnen Fallberichten aufzudecken, kaum möglich ist.

In den wenigen bisher durchgeführten systematischen Untersuchungen zeigte sich, dass Menschen mit einer Autismus-Spektrum-Störung, die eine Sexualstraftat begangen haben, in ihrer Kindheit häufiger emotionalen und physischen Missbrauch und Vernachlässigung erfahren haben, häufig bereits im Kindes- und Jugendalter auffälliges sexuelles Verhalten gezeigt haben (u. a. exzessives Masturbieren, Masturbieren bzw. das Entblößen der eigenen Genitalien an öffentlichen Plätzen), häufiger kognitive und intellektuelle Beeinträchtigungen aufweisen sowie seltener eine ausreichende sexuelle Aufklärung und Erziehung erhalten haben. Darüber hinaus stellen sexuelle Übergriffe, begangen von Menschen mit einer Autismus-Spektrum-Störung, eher impulsive Handlungen dar (Søndenaa et al. 2014).

In der Literatur finden sich einzelne Erklärungsansätze über einen möglichen Zusammenhang zwischen einer $\mathrm{Au}$ tismus-Spektrum-Störung und sexuell übergriffigem Verhalten, die jedoch hauptsächlich von Fallstudien abgeleitet wurden:

- Die übermäßige Fixierung auf bestimmte Interessen (z.B. Pornographie) oder bestimmte Körpermerkmale (z.B. Haare, Schuhe) könnte über die Entwicklung einer hypersexuellen Störung bzw. einer paraphilen Störung einen Risikofaktor für die Begehung einer Sexualstraftat darstellen (Brendel et al. 2002; Chan und Saluja 2011; Haskins und Silva 2006).

- Die fehlenden bzw. herabgesetzten sozialen und kommunikativen Fähigkeiten könnten die Initiierung einer auf Gegenseitigkeit beruhenden sexuellen Beziehung erschweren, sodass auf andere Wege, u. a. sexuelle Gewalt, zurückgegriffen werden muss (Griffin-Shelley 2010).

- Die fehlenden oder nur gering ausgeprägten Theory-ofMind-Fähigkeiten könnten eine herabgesetzte Empathie für die Opfer von Sexualstraftaten bewirken, was die eigene Hemmschwelle für die Begehung einer Sexualstraftat herabsetzen könnte (Murrie et al. 2002).

- Ein reduziertes Verständnis für soziale Normen könnte zu mehr kognitiven Verzerrungen führen, die ebenfalls einen prognostisch ungünstigen Faktor für sexuelle Delinquenz darstellen (Milton et al. 2002). 
- Durch die nicht auf die Besonderheiten von Menschen mit einer Autismus-Spektrum-Störung zugeschnittene sexuelle Aufklärung und Erziehung fehlen basales Wissen über und Fähigkeiten zu strafrechtskonformen sexuellen Verhaltensweisen (Schöttle et al. 2017).

Auch wenn diese Erklärungsansätze bisher empirisch nicht hinreichend untersucht wurden, so stellen sie doch einen möglichen Ausgangspunkt für therapeutische Interventionen mit Menschen mit einer Autismus-SpektrumStörung, die eine Sexualstraftat begangen haben, dar. Es wurde vorgeschlagen, dass psychotherapeutische Interventionen im Gegensatz zu Therapieprogrammen mit nichtautistischen Sexualstraftätern eher im Einzelsetting durchgeführt werden sollten (Sutton et al. 2013). In der Einzeltherapie sollten in Abhängigkeit des intellektuellen Leistungsniveaus sowie der von den oben genannten Erklärungsansätzen abgeleiteten vorliegenden individuellen Defizite u. a. folgende Themengebiete bearbeitet werden: Aufbau eines ausreichenden Wissens über Sexualität, Aufbau strafrechtskonformer sexueller Verhaltensweisen, soziales Kompetenztraining mit dem Schwerpunkt der Verbesserung der verbalen und nonverbalen Kommunikationsfähigkeiten, Aufbau eines ausreichenden Empathieempfindens, Bearbeitung kognitiver Verzerrungen und eine Verbesserung der Impulskontrolle (Allely und Creaby-Attwood 2016). Aufgrund des in einigen Fallberichten beschriebenen suchtartigen Charakters sexuellen Verhaltens sowie der teilweise ganz erheblichen Einschränkungen der intellektuellen Leistungsfähigkeit ist es außerdem vorstellbar, dass manche Sexualstraftäter mit einer Autismus-Spektrum-Störung insbesondere von einer medikamentösen, triebdämpfenden Therapie profitieren könnten (Turner et al. 2014; Turner und Briken 2018).

\section{Forensisch-psychiatrische Begutachtung}

International gibt es Autoren, die einen Einfluss der autismusspezifischen Symptome auf die Fähigkeit zur Urteilsbildung sowie auf die Selbstkontrolle diskutieren, die in ausgewählten Fällen auch Bedeutung für die Beurteilung der Einsichts- und Steuerungsfähigkeit im Rahmen der Schuldfähigkeitsbegutachtung innerhalb des deutschen Strafrechtssystems haben könnte. Eine direkte Übertragung auf die Schuldfähigkeitsbegutachtung in Deutschland sollte aufgrund der bestehenden Unterschiede zwischen den einzelnen Rechtssystemen aber unbedingt vermieden werden, dennoch kann die Auseinandersetzung mit der internationalen Literatur einige Denkanstöße liefern.

Katz und Zemishlany (2006) beschrieben, dass eine autistische Entwicklung zu einer psychoseähnlichen Einengung der Kognitionen und Emotionen sowie einem fehler- haften schlussfolgernden Denken führen könnte. Verbunden mit den deutlichen Defiziten, soziale Situationen zu erfassen und den Standpunkt des Gegenübers einzunehmen, könnte dies, zumindest im Rahmen des israelischen Strafrechts, eine reduzierte Fähigkeit, das Unrecht einer Tat einzusehen, bedingen (Katz und Zemishlany 2006). In einer aktuellen Übersichtsarbeit, die sich auf das kanadische Rechtssystem bezieht, wurde dargestellt, dass die ausgeprägten Empathiedefizite bei Menschen mit einer Autismus-Spektrum-Störung zum einen zu einem fehlerhaften Verständnis der Konsequenzen der eigenen Handlungen und zum anderen zu Fehlinterpretationen des Verhaltens und psychischen Befindens des Gegenübers führen könnten (z.B. das Gegenüber wird fälscherweise als bedrohlich eingestuft, und die strafrechtlich relevante Handlung der Person mit einer Autismus-Spektrum-Störung stellt eine Reaktion auf die fälschlicherweise wahrgenommene Bedrohung dar) (Grant et al. 2018). Umgekehrt könnte sich das rigide Haften an und die unflexible Interpretation von moralischen Wertvorstellungen bei Menschen mit einer Autismus-Spektrum-Störung in einem strikten Festhalten an und einem apodiktischen Beharren auf den sozialen Normen des Rechtsstaates äußern, was letztlich sogar einen protektiven Faktor bezüglich zukünftiger Straffälligkeit darstellen könnte (Lindsay et al. 2014; Grant et al. 2018). Komplexere soziale Situationen, in denen eine flexible Anpassung der eigenen moralischen Vorstellungen und Einstellungen nötig ist, könnten jedoch zu einem Zusammenbrechen der strikten moralischen Wertvorstellungen führen, was sich im individuellen Fall in impulsiv-straffälligem Verhalten des Betroffenen äußern könnte (Grant et al. 2018).

In Deutschland lässt sich ein Asperger-Syndrom bzw. eine hochfunktionale Autismus-Spektrum-Störung im Rahmen der Schuldfähigkeitsbegutachtung u.U. dem vierten Eingangsmerkmal nach dem $\S 20$ StGB, der ,schweren anderen seelischen Abartigkeit", zuordnen. Bei Personen mit einem frühkindlichen oder atypischen Autismus muss in Abhängigkeit des intellektuellen Leistungsniveaus auch das Vorliegen des dritten Eingangsmerkmals, des „Schwachsinns“, diskutiert werden. Die Autoren der aktuellen deutschsprachigen forensisch-psychiatrischen Lehrbücher empfehlen, bei einem Straftäter mit der Diagnose einer Autismus-Spektrum-Störung sowie einer zumindest durchschnittlichen intellektuellen Leistungsfähigkeit (Asperger-Syndrom bzw. hochfunktionalem Autismus) die Einsichtsfähigkeit in der Regel als gegeben anzusehen (Günter 2015). Auch die Feststellung einer erheblichen Beeinträchtigung der Steuerungsfähigkeit sollte eher zurückhaltend angewendet werden (Günter 2015). Dennoch sollte beispielsweise bei Straftaten, die sich aus einem Unverständnis der sozialen Gegebenheiten ableiten lassen (z.B. ein Proband mit einer Autismus-Spektrum-Störung missinterpretiert das Verhalten seines Gegenübers als be- 
leidigend bzw. als Angriff gegen die eigene Person und reagiert daher aggressiv und gewalttätig) oder als Folge des starren und stereotypen Festhaltens an bestimmten Routinen entstehen (z.B. ein routinemäßiger Handlungsablauf wird durch ein unerwartetes Ereignis gestört, und der Proband reagiert mit deutlicher innerer Unruhe und einem psychomotorischen Erregungszustand, in dem eine andere Person verletzt wird) eine Beeinträchtigung der Steuerungsfähigkeit zumindest diskutiert werden (Günter 2012, 2015; Remschmidt 2016). Wie in allen Fällen ist jedoch eine individualisierte Vorgehensweise bei der forensisch-psychiatrischen Begutachtung notwendig, um dem Einzelfall gerecht zu werden. Dies insbesondere vor dem Hintergrund der häufig komorbid auftretenden psychischen Erkrankungen, die ebenfalls einen Einfluss auf mögliche Straffälligkeit ausüben könnten (Palermo 2004; Remschmidt 2016).

Abschließend kann festgehalten werden, dass der aktuelle Forschungsstand bezüglich des Zusammenhangs zwischen Straffälligkeit und einer Autismus-Spektrum-Störung weiterhin sehr überschaubar ist. Zukünftige Untersuchungen sollten sich daher insbesondere auf den Zusammenhang der störungsspezifischen Symptome (z. B. repetitive Interessen, Hypo- oder Hypersensibilitäten, fehlende Theory-ofMind-Fähigkeiten oder kognitive Verzerrungen) und Straffälligkeit konzentrieren. Dadurch können wichtige Erkenntnisse nicht nur bezüglich einer deliktpräventiven und störungsspezifischen Therapie, sondern auch bezüglich der forensisch-psychiatrischen Begutachtung gewonnen werden.

Funding Open Access funding enabled and organized by Projekt DEAL.

Interessenkonflikt D. Turner, D. Schöttle und P. Briken geben an, dass kein Interessenkonflikt besteht.

Open Access Dieser Artikel wird unter der Creative Commons Namensnennung 4.0 International Lizenz veröffentlicht, welche die Nutzung, Vervielfältigung, Bearbeitung, Verbreitung und Wiedergabe in jeglichem Medium und Format erlaubt, sofern Sie den/die ursprünglichen Autor(en) und die Quelle ordnungsgemäß nennen, einen Link zur Creative Commons Lizenz beifügen und angeben, ob Änderungen vorgenommen wurden.

Die in diesem Artikel enthaltenen Bilder und sonstiges Drittmaterial unterliegen ebenfalls der genannten Creative Commons Lizenz, sofern sich aus der Abbildungslegende nichts anderes ergibt. Sofern das betreffende Material nicht unter der genannten Creative Commons Lizenz steht und die betreffende Handlung nicht nach gesetzlichen Vorschriften erlaubt ist, ist für die oben aufgeführten Weiterverwendungen des Materials die Einwilligung des jeweiligen Rechteinhabers einzuholen.

Weitere Details zur Lizenz entnehmen Sie bitte der Lizenzinformation auf http://creativecommons.org/licenses/by/4.0/deed.de.

\section{Literatur}

Allely CS, Creaby-Attwood A (2016) Sexual offending and autism spectrum disorders. Jnl Intell Disabil Offen Behav 7(1):35-51
American Psychiatric Association (2013) Diagnostic and statistical manual of mental disorders (DSM-5). American Psychiatric Publishing, Washington, DC

Bölte S, Poustka F, Rühl D, Schmötzer G (2006) Diagnostisches Interview für Autismus - Revidiert. Hogrefe, Göttingen

Brendel DH, Bodkin JA, Hauptman B, Ornstein A (2002) "I See Dead People": Overcoming psychic numbness. Harv Rev Psychiatry 10(3): 166-178

Byers ES, Nichols S, Voyer SD (2013a) Challenging stereotypes: sexual functioning of single adults with high functioning autism spectrum disorder. J Autism Dev Disord 43:2617-2627. https://doi. org/10.1007/s10803-013-1813-z

Byers ES, Nichols S, Voyer SD, Reilly G (2013b) Sexual well-being of a community sample of high-functioning adults on the autism spectrum who have been in a romantic relationship. Autism 17(4):418-433. https://doi.org/10.1177/1362361311431950

Chan LG, Saluja B (2011) Sexual offending and improvement in autistic characteristics after acquired brain injury: a case report. Aust N Z J Psychiatry 45(10):902-903

Clements J, Zarkowska E (2000) Behavioral concerns and autistic spectrum disorders: Explanations and strategies for change. Jessica Kingsley Publishers, London

Dean M, Harwood R, Kasari C (2017) The art of camouflage: Gender differences in the social behaviors of girls and boys with autism spectrum disorder. Autism 21(6):678-689

Fernandes LC, Gillberg CI, Cederlund M, Hagberg B, Gillberg C, Billstedt E (2016) Aspects of sexuality in adolescents and adults diagnosed with autism spectrum disorders in childhood. J Autism Dev Disord 46(9):3155-3165

Fombonne E (2009) Epidemiology of pervasive developmental disorders. Pediatr Res 65(6):591-598. https://doi.org/10.1203/PDR. 0b013e31819e7203

Glidden D, Bouman WP, Jones BA, Arcelus J (2016) Gender dysphoria and autism spectrum disorder: a systematic review of the literature. Sex Med Rev 4(1):3-14. https://doi.org/10.1016/j.sxmr. 2015.10.003

Grant T, Furlano R, Hall L, Kelley E (2018) Criminal responsibility in autism spectrum disorder: a critical review examining empathy and moral reasoning. Can Psychol 59(1):65-75. https://doi.org/ $10.1037 /$ cap0000124

Griffin-Shelley E (2010) An Asperger's adolescent sex addict, sex offender: a case study. Sex Addict Compulsivity 17(1):46-64

Günter M (2012) Dissozialität bei Jugendlichen mit Asperger-Syndrom - Ausdruck von Normalität oder Folge des Erlebens von Ausgrenzung und Fremdheit? Forens Psychiatr Psychother $8(1): 5-17$

Günter M (2015) Strafrechtliche Begutachtung von Jugendlichen und Heranwachsenden. In: Dreßing H, Habermeyer E (Hrsg) Psychiatrische Begutachtung: Ein praktisches Handbuch für Ärzte und Juristen. Elsevier Urban \& Fischer, München, S 579-605

Haskins BG, Silva JA (2006) Asperger's disorder and criminal behavior: Forensic-psychiatric considerations. J Am Acad Psychiatry Law 34:374-384

Hellemans H, Colson K, Verbraeken C, Vermeiren R, Deboutte D (2007) Sexual behavior in high-functioning male adolescents and young adults with autism spectrum disorder. J Autism Dev Disord 37(2):260-269. https://doi.org/10.1007/s10803-006-0159-1

Katz N, Zemishlany Z (2006) Criminal responsibility in Asperger's Syndrome. Isr J Psychiatry Relat Sci 43(3):166-173

King C, Murphy GH (2014) A systematic review of people with autism spectrum disorder and the criminal justice system. J Autism Dev Disord 44(11):2717-2733

Koller R (2000) Sexuality and adolescents with autism. Sex Disabil 18(2):125-135

Konstantareas MM, Lunsky YJ (1997) Sociosexual knowledge, experience, attitudes, and interests of individuals with autistic disorder and developmental delay. J Autism Dev Disord 27(4):397-413 (http://www.ncbi.nlm.nih.gov/pubmed/9261666) 
Lindsay WR, Carson D, O’Brien G, Holland AJ, Taylor JL, Wheeler JR, Steptoe L (2014) A comparison of referrals with and without Autism Spectrum Disorder to forensic intellectual disability services. Psychiatry Psychol Law 21(6):947-954

Matthies S, Frauenknecht S (2019) Autismus und ADHS im Erwachsenenalter. In: Lieb K, Frauenknecht S (Hrsg) Intensivkurs Psychiatrie und Psychotherapie. Elsevier, München, S 463-472

Milton J, Duggan C, Latham A, Egan V, Tantam D (2002) Case history of co-morbid Asperger's syndrome and paraphilic behaviour. Med Sci Law 42(3):237-244. https://doi.org/10.1177/ 002580240204200308

Mouridsen SE (2012) Current status of research on autism spectrum disorders and offending. Res Autism Spectr Disord 6(1):79-86

Murrie DC, Warren JI, Kristiansson M, Dietz PE (2002) Asperger's syndrome in forensic settings. Int J Forensic Ment Health 1(1):59-70. https://doi.org/10.1080/14999013.2002.10471161

Oien RA, Cicchetti DV, Nordahl-Hansen A (2018) Gender dysphoria, sexuality and autism spectrum disorders: a systemativ map review. J Autism Dev Disord 48(12):4028-4037

Palermo MT (2004) Pervasive developmental disorders, psychiatric comorbidities and the law. Int $\mathrm{J}$ Offender Ther Comp Criminol 48:40-48

Pellicano E (2013) Sensory symptoms in autism: a blooming, buzzing confusion? Child Dev Perspect 7(3): 143-148

Poustka L, Rühl D, Feineis-Matthews S, Poustka F, Hartung M, Bölte S (2015) Diagnostische Beobachtungsskala für Autistische Störungen. Hogrefe, Göttingen

Reid RC, Garos S, Carpenter BN (2011) Reliability, validity, and psychometric development of the hypersexual behavior inventory in an outpatient sample of men. Sex Addict Compulsivity 18(1):30-51. https://doi.org/10.1080/10720162.2011.555709

Remschmidt H (2016) Entwicklungsstörungen. In: Häßler F, Kinze W, Nedopil N (Hrsg) Praxishandbuch Forensische Psychiatrie: Grundlagen, Begutachtung, Interventionen im Erwachsenen-, Jugendlichen- und Kindesalter. Medizinisch Wissenschaftliche Verlagsgesellschaft, Berlin, S 361-380

Schöttle D, Briken P, Tüscher O, Turner D (2017) Sexuality in autism: hypersexual and paraphilic behavior in women and men with high-functioning autism spectrum disorder. Dialogues Clin Neurosci 19(4):381-393. https://doi.org/10.1038/jid.2015.269

Shaeer O, Sheer K (2015) The global online sexuality survey: the United States of america in 2011: homosexuality among Englishspeaking men. Hum Androl 5(3):45-48
Søndenaa E, Rasmussen K, Helverschou SB, Steindal K, Nilson B, Nøttestad JA (2014) Violence and sexual offending behavior in people with autism spectrum disorder who have undergone a psychiatric forensic examination. Psychol Rep 115(1):32-43. https:// doi.org/10.2466/16.15.PR0.115c16z5

Strunz S, Schermuck C, Ballerstein S, Ahlers CJ, Dziobek I, Roepke $S$ (2017) Romantic relationships and relationship satisfaction among adults with Asperger syndrome and high-functioning autism. J Clin Psychol 73(1):113-125https://doi.org/10.1002/jclp. 22319

Sutton LR, Hughes TL, Huang A, Lehman C, Paserba D, Talkington V, Marshall S et al (2013) Identifying individuals with autism in a state facility for adolescents adjudicated as sexual offenders: a pilot study. Focus Autism Other Dev Disabl 28(3):175-183

Turner D, Briken P (2017) Wenn unkontrollierbares sexuelles Verhalten negative Folgen hat. Diagnostik und Therapie der hypersexuellen Störung. InFo Neurol Psychiatr 19(12):28-36

Turner D, Briken P (2018) Treatment of paraphilic disorders in sexual offenders or men with a risk of sexual offending with Luteinizing hormone-releasing hormone agonists: an updated systematic review. J Sex Med 15(1):77-93. https://doi.org/10.1016/j.jsxm. 2017.11.013

Turner D, Basdekis-Jozsa R, Dekker A, Briken P (2014) Which factors infl uence the appropriateness of testosterone-lowering medications for sex offenders? A survey among clinicians from German forensic-psychiatric institutions. World J Biol Psychiatry 15(6):472-478. https://doi.org/10.3109/15622975.2013.839052

Turner D, Briken P, Schöttle D (2017) Autism-spectrum disorders in adolescence and adulthood: focus on sexuality. Curr Opin Psychiatry 30(6):409-416. https://doi.org/10.1097/YCO. 0000000000000369

Turner D, Briken P, Schöttle D (2019) Sexual dysfunctions and their association with the dual control model of sexual response in men and women with high-functioning autism. JCM 8(4):425. https:// doi.org/10.3390/jcm8040425

Woodbury-Smith MR, Clare ICH, Holland AJ, Kearns A (2006) High functioning autistic spectrum disorders, offending and other lawbreaking: findings from a community sample. J Forensic Psychiatry Psychol 17(1):108-120

World Health Organization (1993) The International Classification of Diseases-10. World Health Organization, Geneva 